\title{
Preoperative full-length standing radiographs and revision rates in lumbar degenerative scoliosis
}

\author{
Joshua T. Bunch, MD, ${ }^{1}$ Steven D. Glassman, MD, ${ }^{1}$ Howard R. Underwood, MD, MBA, MS, ${ }^{2}$ \\ Leanne N. Metcalfe, PhD, ${ }^{2}$ Stephen Ondra, MD, ${ }^{2}$ Ivan Vasilyev, MS, ${ }^{2}$ and \\ Leah Y. Carreon, MD, MSc ${ }^{1}$
}

${ }^{1}$ Norton Leatherman Spine Center, Louisville, Kentucky; and ${ }^{2 H e a l t h ~ C a r e ~ S e r v i c e ~ C o r p o r a t i o n, ~ E n t e r p r i s e ~ C l i n i c a l ~ A n a l y t i c s, ~}$ Chicago, Illinois

OBJECTIVE Full-length (36-inch) standing spine radiographs are commonly used by spine surgeons to evaluate patients with lumbar degenerative scoliosis (LDS). Despite this practice, the impact of these images on preoperative decision making and the rate of revision surgery has not been analyzed. The purpose of this study is to determine if preoperative full-length standing spine radiographs improve surgical decision making by decreasing the rate of revision surgery in patients with LDS.

METHODS From the Health Care Service Corporation administrative claims database, the authors identified patients $50-80$ years of age with LDS who had undergone surgery including posterior lumbar decompression and fusion over 2-6 levels and with at least 5 years of continuous coverage after the index surgery. Patients were stratified into the following groups, according to the preoperative imaging studies performed within 6 months before their index surgery: lumbar spine MRI studies only, lumbar spine MRI studies and standard lumbar spine radiographs, CT myelograms, and full-length standing spine radiographs. Survival analysis was performed with the occurrence of a revision within 5 years of the index surgery as the outcome of interest.

RESULTS A total of 411 patients were included in the study after applying the inclusion and exclusion criteria. Revision surgery within 5 years after the index procedure was most frequent in the patients with preoperative MRI only (41.8\%), followed by the patients with a CT myelogram $(30.4 \%)$ and those with MRI and standard radiographs (24.8\%). The lowest revision rate was seen among those with long-cassette standing radiographs (11.1\%). Patients whose preoperative evaluation included full-length standing radiographs (OR 0.353, $p=0.034)$ and MRI studies plus radiographs $(O R$ 0.650, $p=0.022$ ) were less likely to require revision surgery at 5 years after the index procedure.

CONCLUSIONS An assessment of standing alignment using full-length (36-inch) standing radiographs may be beneficial in reducing the risk of revision surgery in patients with lumbar scoliosis. This observation was not limited to patients with large curves or substantial deformity.

https://thejns.org/doi/abs/10.3171/2017.10.SPINE17638

KEYWORDS lumbar degenerative scoliosis; preoperative planning; revision surgery

A DULT lumbar degenerative scoliosis (LDS) refers to a coronal deformity of the lumbar spine (lumbar Cobb measurement $>10^{\circ}$ ) that is often associated with spondylolisthesis, stenosis, loss of lordosis, axial rotation, and lateral listhesis., ${ }^{2,14}$ It can form de novo, as an individual ages, or as a consequence of childhood scoliosis. ${ }^{2}$ Associated degenerative changes can include facet hypertrophy, thickening of the ligamentum flavum, asymmetri- cal disc collapse, and disc herniation. ${ }^{11,14}$ Conceptually, individuals with adult-onset LDS can often be divided into 2 broad groups: those with deformity-related symptoms and those with symptomatic stenosis. Patients with symptoms attributable to the deformity itself generally have larger curves, often with structural sagittal malalignment. These patients may require major realignment procedures. A second, much more common group of adults with LDS

ABBREVIATIONS AUC = appropriate use criteria; $\mathrm{HCSC}=$ Health Care Service Corporation; $\mathrm{LDS}=$ lumbar degenerative scoliosis.

SUBMITTED June 8, 2017. ACCEPTED October 11, 2017.

INCLUDE WHEN CITING Published online March 23, 2018; DOI: 10.3171/2017.10.SPINE17638. 
present with symptomatic spinal stenosis in association with smaller degenerative curves and may require surgery primarily for stenosis. ${ }^{5}$

As LDS presents in numerous patterns, the array of possible treatment options is also quite variable. ${ }^{13}$ In an effort to provide evidence-based guidance for the care of patients with LDS, an appropriate use criteria (AUC) evaluation of LDS was performed by RAND/UCLA. ${ }^{2,5}$ The LDS AUC outline numerous clinical scenarios and the treatment considered appropriate, inappropriate, or indeterminate for each of these scenarios.

An underlying tenet set forth in the LDS AUC was that weight-bearing spine radiographs, ideally full-length (36inch) standing radiographs, be obtained as a prerequisite when evaluating any patient with LDS for surgical treatment. ${ }^{2,5}$ This stipulation of the AUC is based on panelist recommendations but has not been demonstrated in the literature. Therefore, the objective of this study was to determine if preoperative full-length standing spine radiographs improve surgical decision making, thus decreasing the rate of revision spine surgery in patients with LDS. In particular, we sought to examine cases with more limited scoliosis such that a standard spinal deformity evaluation might not be performed.

\section{Methods}

From the Health Care Service Corporation (HCSC) administrative claims database, we identified patients 50-80 years of age with LDS who had undergone surgical treatment including posterior lumbar decompression and fusion over 2-6 spinal levels. All patients had a diagnosis of lumbar scoliosis within 6 months of the index surgery, although this was not necessarily the diagnosis assigned to the index surgical intervention. This was done by design so that patients with smaller degenerative curves would not be excluded as these patients are often operated on for diagnoses other than deformity, such as stenosis. Additionally, all patients had at least 5 years of continuous coverage in the HCSC system after the index surgery. Exclusion criteria were postlaminectomy kyphosis, posterior fusions of more than 6 levels, anterior fusions during the same admission as the index procedure or within 14 days of the index procedure, and patients who had undergone a spinal osteotomy. These additional criteria were applied to exclude cases of complex adult spinal deformity (Table 1).

Patients were then stratified into the following groups, based on the preoperative imaging performed within 6 months before the index surgery: lumbar MRI studies only, MRI studies of the lumbar spine and standard lumbar spine radiographs, CT myelograms, and full-length standing spine radiographs. Fisher's exact test was used to compare the number of cases requiring revision surgery within 5 years after the index procedure. Survival analysis was performed with the occurrence of a revision within 5 years of the index surgery as the outcome of interest. Irrigation and debridements were not included as revision procedures. After the initial survival analysis, a regression analysis was performed using data at the 3-year and 5-year follow-up time points to determine factors associated with the occurrence of a revision surgery.

\section{Results}

A total of 411 patients met inclusion criteria. Six hundred twenty-two patients had anterior procedures during the same admission or within 14 days of the admission and were excluded, 432 patients were excluded because of long posterior fusions, and 11 patients had spinal osteotomies and were therefore excluded. Two hundred fifty-four individuals did not have adequate 5-year follow-up and were excluded. The sex distribution was similar among the 4 patient groups (Table 2). The MRI-only group was older than the 3 other groups. The need for revision surgery within 5 years after the index surgery was highest in the patients with MRI only (41.8\%). Patients with CT myelograms $(30.4 \%)$ or MRI and standard radiographs $(24.8 \%)$ had similar revision rates. Patients with long-cassette standing radiographs preoperatively had the lowest rate of revision surgery within 5 years of the index procedure $(11.1 \%)$. The trend was similar when examining the number of revision surgeries performed (Table 3 ).

TABLE 1. Study population

\begin{tabular}{ll}
\hline \multicolumn{1}{c}{ Parameter } & Description (ICD-9-CM \& CPT codes) \\
\hline Demographics & Include patients btwn 50 \& 80 yrs old \\
\hline Diagnoses & Include patients w/ degenerative scoliosis, stenosis $(724.02), \&$ scoliosis $(737.39,737.40,737.41,737.42$, \\
& $737.43,737.80,738.50)$ codes w/in 6 mos of index surgery, not necessarily concurrent or w/in same visit \\
& Exclude patients w/ postlaminectomy kyphosis $(722.83)$ diagnosed prior to index surgery \\
\hline Procedures & Include index surgery: pst fusion of 2-6 levels $(81.07,22842,22800,22634,22614,22840,22630,2263)$ \\
& Include index surgery: pst decompressions $(63005,63012,63017,63030,63035,63047,63048)$ \\
& Exclude ant fusions $(81.04,81.06,22558,22585,22808,22810,22812,22845,22846,22847)$ performed \\
& during same admission or w/in 14 days of pst fusion of 2-6 levels or decompression \\
& Exclude long pst fusions $(81.05,22843,22844,22804,22802)$ \\
& Exclude osteotomies $(22206,22207,22208,22212,22214,22216)$ \\
\hline Interventions & Lumbar MRI w/in 6 mos before index surgery $(72148,72149,72158)$ \\
& Short radiographs w/in 6 mos before index surgery $(72100,72110,72114,72120)$ \\
& Long radiographs w/in 6 mos before index surgery $(72010,72090)$ \\
& CT myelogram w/in 6 mos before index surgery $(72255,72129,72265,72132,62284)$ \\
\hline
\end{tabular}

Ant = anterior; $\mathrm{CPT}=$ Current Procedural Terminology; pst = posterior. 
TABLE 2. Summary of demographic data

\begin{tabular}{lccccc}
\hline Parameter & MRI Only & MRI \& Short Radiographs & CT Myelograms & Long Radiographs & $p$ Value \\
\hline Sample size & 55 & 250 & 79 & 27 & \\
\hline Age & 68.15 & 65.38 & 66.2 & 66.37 & $<0.000$ \\
\hline Male sex & $21(38.2 \%)$ & $83(33.2 \%)$ & $30(38.0 \%)$ & $5(18.5 \%)$ & 0.268 \\
\hline
\end{tabular}

Results of the survival analysis based on the type of preoperative imaging are shown in Fig. 1. At 5 years after the index procedure, patients with preoperative lumbar radiographs and long-cassette standing radiographs were less likely to require revision surgery. The OR associated with revision surgery for patients with pre-index surgery long radiographs was 0.353 (95\% CI $0.135-0.924$, $\mathrm{p}=0.034)$. The OR for revision surgery for patients with standing lumbar radiographs was 0.650 (95\% CI 0.450 $0.939, \mathrm{p}=0.022$ ).

\section{Discussion}

The presentation of LDS varies widely, and this may contribute to the variability in treatment patterns. Such variability in management offers an opportunity to optimize care through the development and implementation of evidence-based treatment. The development of AUC was undertaken to fill this need, and the criteria outline the management of LDS with recommendations for optimal procedure selection given the type and severity of pathology being treated. The expert panel involved in developing the AUC identified adequate preoperative imaging with weight-bearing spine radiographs, ideally full-length (36-inch) standing radiographs, as a prerequisite for the application of these guidelines. ${ }^{2,5}$

Spinal deformity assessment including 36-inch standing radiographs is standard in patients with substantial deformity. Numerous studies have previously used full-length standing radiographs to assess spinal alignment, $4,7,8,15,16$ and the Scoliosis Research Society adult deformity classification is based on full-length standing radiographs. ${ }^{9} \mathrm{On}$ the other hand, patients with less deformity and primary neurogenic complaints are evaluated in a less standardized fashion. In this group, in particular, the impact of preoperative standing radiographs on surgical decision making in LDS has not been evaluated in the literature.
In the present study, the rate of revision surgery at 5 years postoperatively was significantly lower in patients who had full-length standing spine radiographs than in those who did not. The advantage of preoperative longcassette standing radiographs in preoperative planning is also demonstrated by the multivariate logistic regression analysis that showed a significantly lower likelihood of revision surgery at 5 years than in those without longcassette standing radiographs.

Patients with only preoperative MRI had the highest rate of revision surgery. This may reflect a failure to appreciate significant coronal and/or sagittal malalignment or a failure to account for weight-bearing-accentuated spinal stenosis as part of the preoperative planning process. Historically, this may have been addressed by the more frequent use of CT myelography in scoliosis patients. The revision surgery rate was somewhat lower in patients with MRI and standard lumbar radiographs as well as in those with CT myelograms; both of these neuroimaging modalities at least partially account for the weight-bearing changes characteristically seen in LDS. Importantly, these data suggest that the addition of relatively inexpensive 36-inch radiographs should be dominant from a cost-effectiveness standpoint in that such imaging studies both improve care and reduce costs associated with reoperation.

The impact of the routine use of 36-inch radiographs on improving outcomes and decreasing costs in the treatment of patients with LDS is not completely clear, but the existing literature suggests it may be substantial. Curve magnitude has been found to be inversely proportional to curve prevalence, so most surgical procedures involve smaller curves. ${ }^{1,3}$ Several surgical series have reported on the treatment of lumbar curves averaging in the $20^{\circ}$ range. ${ }^{3,6,10}$ Additionally, Schwab et al. reported a mean curve magnitude of only $25^{\circ}$ among a patient population with de novo degenerative scoliosis. ${ }^{12}$ These individuals with smaller deformities are the patients who seemingly

TABLE 3. Incidence of revision surgery per year stratified by imaging used

\begin{tabular}{|c|c|c|c|c|c|}
\hline Parameter & MRI Studies Only & MRI \& Short Radiographs & CT Myelograms & Long Radiographs & $\mathrm{p}$ Value \\
\hline Sample size & 55 & 250 & 79 & 27 & \\
\hline \multicolumn{6}{|l|}{ Revision surgery } \\
\hline $1 \mathrm{yr}$ & 6 & 15 & 4 & 3 & \\
\hline $2 \mathrm{yrs}$ & 2 & 22 & 9 & 2 & \\
\hline 3 yrs & 4 & 17 & 7 & 0 & \\
\hline 4 yrs & 9 & 12 & 3 & 0 & \\
\hline 5 yrs & 8 & 8 & 5 & 0 & \\
\hline Total revisions & $29(52.7 \%)$ & $74(29.6 \%)$ & $28(35.4 \%)$ & $5(18.5 \%)$ & \\
\hline Cases requiring revision & $23(41.8 \%)$ & $62(24.8 \%)$ & $24(30.4 \%)$ & $3(11.1 \%)$ & 0.014 \\
\hline
\end{tabular}




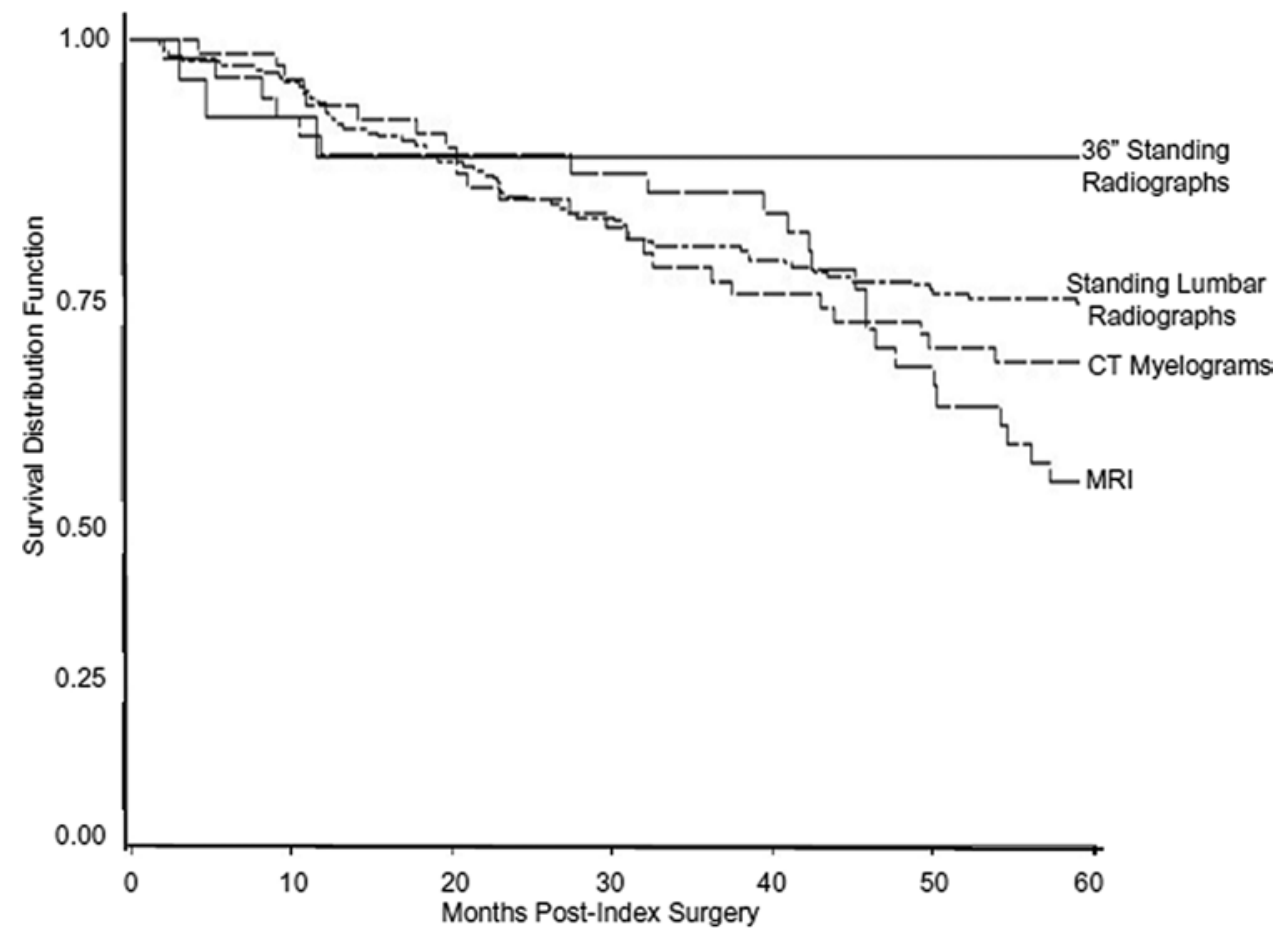

FIG. 1. Kaplan-Meier plot showing the proportion of patients requiring revision surgery after the initial index surgery among the 4 imaging modality cohorts: patients with full-length (36-inch) standing radiographs, standing lumbar radiographs, CT myelograms, and lumbar MRI.

have a more variable preoperative imaging workup and were the target population of our study.

There are significant limitations to the administrative claims-based design of this study. They include the retrospective design, unknown patient comorbidities and curve magnitude, lack of patient-reported outcome measures, and, most importantly, an inability to convey the surgeon's rationale for patient selection or surgical planning. These factors may play an additional role in predicting the rate of revision surgery but could not be assessed in the present study. Additionally, complex adult spinal deformity procedures were not included in this study. Although patients undergoing such procedures represent an important subset of LDS patients, the appropriate preoperative radiographic evaluation of these patients seems more consistent. Lastly, we acknowledge that setting the preoperative imaging window at 6 months represents only one of many possibilities. The optimal time frame for preoperative imaging could not be determined from this data set. Further investigations will be useful in assessing the optimal cutoff point when preoperative imaging should be considered "out of date."

\section{Conclusions}

In summary, this is the first study to evaluate the impact of preoperative imaging on the revision rate in LDS patients. Additionally, this study represents a unique collaborative effort between a tertiary care spine surgery department and a leading insurance provider to address clinical issues relevant to all health care providers, namely the reduction of revision surgery rates and ultimately the reduction of health care costs. The LDS AUC identified weight-bearing spine radiographs as a prerequisite for optimal treatment selection, ${ }^{2,5}$ and our findings validate this presumption. We therefore suggest obtaining full-length (36-inch) standing radiographs in all LDS patients for whom surgical treatment is being considered. We believe that appropriate preoperative imaging, as part of an evidence-based approach set forth in the LDS AUC, will lead to improved management of this challenging and complex patient population.

\section{References}

1. Ailon T, Smith JS, Shaffrey CI, Lenke LG, Brodke D, Harrop JS, et al: Degenerative spinal deformity. Neurosurgery 77 (Suppl 4):S75-S91, 2015

2. Chen PG, Daubs MD, Berven S, Raaen LB, Anderson AT, Asch SM, et al: Surgery for degenerative lumbar scoliosis: the development of appropriateness criteria. Spine (Phila Pa 1976) 41:910-918, 2016

3. Daubs MD, Lenke LG, Bridwell KH, Cheh G, Kim YJ, Stobbs G: Decompression alone versus decompression with limited fusion for treatment of degenerative lumbar scoliosis in the elderly patient. Evid Based Spine Care J 3:27-32, 2012

4. Gelb DE, Lenke LG, Bridwell KH, Blanke K, McEnery KW: An analysis of sagittal spinal alignment in 100 asymptomatic middle and older aged volunteers. Spine (Phila Pa 1976) 20:1351-1358, 1995

5. Glassman SD, Berven SH, Shaffrey CI, Mummaneni PV, Polly DW: Commentary: Appropriate use criteria for lumbar degenerative scoliosis: developing evidence-based guidance for complex treatment decisions. Neurosurgery 80:E205E212, 2017 
6. Hosogane N, Watanabe K, Kono H, Saito M, Toyama Y, Matsumoto M: Curve progression after decompression surgery in patients with mild degenerative scoliosis. J Neurosurg Spine 18:321-326, 2013

7. Jackson RP, McManus AC: Radiographic analysis of sagittal plane alignment and balance in standing volunteers and patients with low back pain matched for age, sex, and size. A prospective controlled clinical study. Spine (Phila Pa 1976) 19:1611-1618, 1994

8. Lafage V, Schwab F, Skalli W, Hawkinson N, Gagey PM, Ondra S, et al: Standing balance and sagittal plane spinal deformity: analysis of spinopelvic and gravity line parameters. Spine (Phila Pa 1976) 33:1572-1578, 2008

9. Lowe T, Berven SH, Schwab FJ, Bridwell KH: The SRS classification for adult spinal deformity: building on the King/ Moe and Lenke classification systems. Spine (Phila Pa 1976) 31 (19 Suppl):S119-S125, 2006

10. Park JJ, Carreon LY, Glassman SD: Adult lumbar degenerative scoliosis $40^{\circ}$ or less: outcomes of surgical treatment with minimum 2-year follow-up. Spine Deform 1:211-216, 2013

11. Ploumis A, Transfledt EE, Denis F: Degenerative lumbar scoliosis associated with spinal stenosis. Spine J 7:428-436, 2007

12. Schwab FJ, Smith VA, Biserni M, Gamez L, Farcy JP, Pagala M: Adult scoliosis: a quantitative radiographic and clinical analysis. Spine (Phila Pa 1976) 27:387-392, 2002

13. Silva FE, Lenke LG: Adult degenerative scoliosis: evaluation and management. Neurosurg Focus 28(3):E1, 2010

14. Tribus CB: Degenerative lumbar scoliosis: evaluation and management. J Am Acad Orthop Surg 11:174-183, 2003

15. Van Royen BJ, Toussaint HM, Kingma I, Bot SD, Caspers M, Harlaar J, et al: Accuracy of the sagittal vertical axis in a standing lateral radiograph as a measurement of balance in spinal deformities. Eur Spine J 7:408-412, 1998
16. Vedantam R, Lenke LG, Keeney JA, Bridwell KH: Comparison of standing sagittal spinal alignment in asymptomatic adolescents and adults. Spine (Phila Pa 1976) 23:211-215, 1998

\section{Disclosures}

Dr. Carreon is an employee of Norton Healthcare, has been a consultant for AOSpine, has received travel funds from the University of Southern Denmark and the University of Louisville, and has received support from Pfizer for non-study-related clinical or research effort. Dr. Glassman is an employee of Norton Healthcare and holds a patent with, has been a consultant for, and has received royalties from Medtronic. NuVasive provided funds directly to the database company; no funds were paid to Dr. Glassman or Norton Healthcare.

\section{Author Contributions}

Conception and design: Glassman, Metcalfe. Acquisition of data: Underwood, Metcalfe, Ondra. Analysis and interpretation of data: Carreon, Bunch, Glassman, Underwood, Metcalfe, Vasilyev. Drafting the article: Bunch. Critically revising the article: Carreon, Bunch, Glassman, Underwood, Metcalfe, Vasilyev. Reviewed submitted version of manuscript: Carreon, Bunch, Glassman, Underwood, Metcalfe, Ondra. Statistical analysis: Ondra, Vasilyev. Administrative/technical/material support: Underwood, Metcalfe, Ondra. Study supervision: Glassman, Metcalfe, Ondra.

\section{Correspondence}

Leah Y. Carreon: Norton Leatherman Spine Center, Louisville, KY.leah.carreon@nortonhealthcare.org. 